\title{
Randomized clinical trial of artemisinin versus non-artemisinin combination therapy for uncomplicated falciparum malaria in Madagascar
}

\author{
Didier Ménard*1, Nohary Nina Harimanana Andrianina1, \\ Zakaherizo Ramiandrasoa ${ }^{1}$, Arthur Randriamanantena ${ }^{2}$, \\ Noéline Rasoarilalao ${ }^{1}$, Martial Jahevitra ${ }^{1}$, Arsène Ratsimbasoa ${ }^{2}$, \\ Luciano Tuseo ${ }^{3}$ and Andrianirina Raveloson ${ }^{4}$
}

\begin{abstract}
Address: ${ }^{1}$ Malaria Unit Research, Institut Pasteur de Madagascar, BP 1274, Antananarivo 101, Madagascar, ${ }^{2}$ Epidemiology Unit, Institut Pasteur de Madagascar, Antananarivo 101, Madagascar, ${ }^{3}$ Roll Back Malaria, WHO Office of Madagascar and La Réunion, Antananarivo 101, Madagascar and ${ }^{4}$ National Malaria Control Programme, Ministry of Health, Antananarivo 101, Madagascar

Email: Didier Ménard* - dmenard@ pasteur.mg; Nohary Nina Harimanana Andrianina - palu@pasteur.mg; Zakaherizo Ramiandrasoa - rajonantenaina@razaka.com; Arthur Randriamanantena - randriaman@yahoo.fr;

Noéline Rasoarilalao - rasoarilalao05@yahoo.fr; Martial Jahevitra - mjahevitra@yahoo.fr; Arsène Ratsimbasoa - arsene@pasteur.mg; Luciano Tuseo - Tuseol@mg.afro.who.int; Andrianirina Raveloson - a_raveloson@hotmail.com

* Corresponding author
\end{abstract}

\section{Published: 22 May 2007}

Malaria Journal 2007, 6:65 doi:10.1 186/1475-2875-6-65

This article is available from: http://www.malariajournal.com/content/6/1/65

(C) 2007 Ménard et al; licensee BioMed Central Ltd.

This is an Open Access article distributed under the terms of the Creative Commons Attribution License (http://creativecommons.org/licenses/by/2.0), which permits unrestricted use, distribution, and reproduction in any medium, provided the original work is properly cited.
Received: 12 January 2007

Accepted: 22 May 2007

\begin{abstract}
Background: Data concerning antimalarial combination treatment for uncomplicated malaria in Madagascar are largely lacking. Randomized clinical trial was designed to assess therapeutic efficacies of chloroquine (CQ), amodiaquine (AQ), sulphadoxine-pyrimethamine (SP), amodiaquine plus sulphadoxine-pyrimethamine combination $(A Q+S P)$ and artesunate plus amodiaquine combination (AQ+AS).

Methods: 287 children between 6 months and 15 years of age, with uncomplicated falciparum malaria, were enrolled in the study. Primary endpoints were the day-14 and day-28 risks of parasitological failure, either unadjusted or adjusted by genotyping.

Results: All treatment regimens, except for CQ treatment, gave clinical cure rates above $97 \%$ by day- 14 and $92 \%$ by day-28 (PCR-corrected). AQ+SP was as effective as AQ+AS. The risk of new infection within the month after therapy was generally higher for $A Q+A S$ than $A Q+S P$.

Conclusion: These findings show that the inexpensive and widely available combination $A Q+S P$ may be valuable in for the treatment of uncomplicated malaria in Madagascar and could have an important role in this country, where much of the drugs administered go to patients who do not have malaria.
\end{abstract}




\section{Background}

Malaria remains one of the most serious health problems worldwide and a leading cause of childhood morbidity and mortality, especially in Africa [1]. At least 300 million clinical cases of malaria occur each year, resulting in more than a million child deaths worldwide. Early diagnosis and prompt effective treatment remain the cornerstone for the reduction of malaria-related morbidity and mortality [2]. However, efforts to control malaria in Africa have been severely compromised by the emergence of resistance in Plasmodium falciparum to the inexpensive and widely used drugs, chloroquine (CQ) and sulphadoxinepyrimethamine (SP) $[3,4]$. As a consequence, the use of combination antimalarial therapy has been widely advocated [5]. Of the available antimalarial drugs, the artemisinins are the most potent and the World Health Organization (WHO) specifically advocates the use of artemisinin-based combination therapy (ACT) as the standard policy for the treatment of uncomplicated falciparum malaria [6]. ACTs give a rapid clinical and parasitological response, may delay the development of resistance and may reduce malaria transmission by killing gametocytes. However, there are concerns regarding the cost and availability of artemisinin-based combination therapy (ACT), and only limited data comparing ACT with other combination therapies in Africa are available [7]. Moreover, it is unclear whether ACTs are appropriate for empirical management of febrile illnesses outside the formal health sector without laboratory confirmation, in the way that CQ and SP have been used for several years.

Malaria treatment in Madagascar is in transition: for over 50 years, malaria was treated successfully using chloroquine (CQ) as first-line treatment. In 2005, the National Malaria Control Programme (NMCP) decided to revise its treatment policy and to replace CQ with artemisininbased combination therapy $(\mathrm{AQ}+\mathrm{AS}$, artesunate plus amodiaquine combination) with the support of resources from the Global Fund. This choice was guided by the recommendations of WHO and data from the most recent clinical trial based on the WHO standard protocol [8]: the trial was conducted on the island of Sainte Marie in 2004 and has demonstrated clinical failure in $36.9 \%$ of cases within two weeks of CQ treatment. Alternatives to ACTs, which include the non-artemisinin-based combination therapy (NACT) amodiaquine plus sulphadoxine/ pyrimethamine $(\mathrm{AQ}+\mathrm{SP})$, were surprisingly not considered, although the WHO's Roll Back Malaria programme had recommended the use of NACT in settings where the efficacy of both component drugs is high; this was the case in Madagascar $[9,10]$.

Few data is available concerning alternative or combinatory antimalarial treatment in uncomplicated malaria in Madagascar. Randomized clinical trial was designed (i) to update knowledge about the therapeutic efficacies of CQ (used for the home management of presumed malaria in children under the age of five years), AQ and SP alone (used for intermittent preventive treatment in pregnant women), (ii) to compare the respective therapeutic efficacies of two combinatory treatments, $\mathrm{AQ}+\mathrm{SP}$ and $\mathrm{AQ}+\mathrm{AS}$, and (iii) to generate data which could be used as a guide for designing a rational antimalarial treatment policy in Madagascar.

\section{Materials and methods Study design and study sites}

The study was conducted between February and June 2006 during and at the end of the rainy season, in primary health centres of Moramanga and Saharevo in the east foothill areas of the Highlands of Madagascar. In this area, malaria transmission is low and predominantly seasonal. The main vector is Anopheles funestus and the number of infective bites associated with Plasmodium falciparum is estimated at $<10$ per person per year. The study protocol was reviewed and approved by the Ethics Committee of the Ministry of Health of Madagascar $\left(\mathrm{N}^{\circ} 007 / \mathrm{SANPF} /\right.$ 2007).

\section{Patients}

Children between six months and 15 years of age presenting at primary health centres of Moramanga and Saharevo were enrolled in the study if they met the following inclusion criteria [11]: (i) monoinfection with $P$. falciparum at a parasitaemia between 1,000 and 200,000/ $\mu \mathrm{l}$, (ii) axillary temperature $\geq 37.5^{\circ} \mathrm{C}$, (iii) body weight $>5 \mathrm{~kg}$, (iv) absence of severe malnutrition, (v) absence of febrile conditions caused by diseases other than malaria, (vi) absence of 'danger signs' (inability to stand, breastfeed or drink; recent convulsions; lethargy or persistent vomiting) and of severe and complicated malaria, (vii) haemoglobin $(\mathrm{Hb}) \geq 5 \mathrm{~g} / \mathrm{dl}$, and (viii) informed written consent of parents/guardians.

Exclusion criteria were: (i) known hypersensitivity to SP, $A Q$, or AS, (ii) detection, during follow-up of mixed malarial infections, and (iii) development of concomitant disease which would interfere with the classification of treatment outcome.

\section{Treatments, randomization and blinding}

Patients were randomly assigned to receive one of five oral therapies: CQ $(10 \mathrm{mg} / \mathrm{kg}$ on days 0 and 1 , and $5 \mathrm{mg} / \mathrm{kg}$ on day 2); AQ (10 mg/kg on days 0,1 , and 2$) ; S P(25 \mathrm{mg} / \mathrm{kg}$ sulphadoxine and $1.25 \mathrm{mg} / \mathrm{kg}$ pyrimethamine as a single dose on day 0$) ; \mathrm{AQ}+\mathrm{SP}$ or AQ + AS ( $4 \mathrm{mg} / \mathrm{kg}$ on days 0 , 1 , and 2). Randomization was performed in blocks of five, and treatment regimens were allocated by an independent individual not involved in the analysis of the study. 
All other study personnel were blinded to the treatment assignments, and patients were not informed of their treatment regimen. Patients were directly observed for 30 minutes after treatment, and the dose was readministered if vomiting occurred. Patients who repeatedly vomited their first dose of study medication were excluded from the study.

\section{Follow-up procedures and classification of treatment outcomes}

Following enrollment, patients were asked to return for follow-up visits on days 1, 2, 3, 7, 14, 21, 28, and any other day if they felt ill. Blood was obtained by finger prick for thick blood smears and storage on filter paper on all follow-up days. Haemoglobin was determined on day 0 and on day 28. Treatment outcomes were classified according to 2003 WHO guidelines as Early Treatment Failure (ETF; danger signs or complicated malaria or failure to adequately respond to therapy on days $0-3$ ), Late Clinical Failure (LCF; danger signs or complicated malaria or fever and parasitaemia on days 4-28 without previously meeting criteria for ETF), Late Parasitological Failure (LPF; asymptomatic parasitaemia on days 4-28 without previously meeting criteria for ETF or LCF), and Adequate Clinical and Parasitological Response (ACPR; absence of parasitaemia on day 28 without previously meeting criteria for ETF, LCF, or LPF) [11].

Patients classified as having suffered treatment failure were treated with quinine $(10 \mathrm{mg} / \mathrm{kg}$ three times daily for 7 days); however, their response to repeat therapy was not assessed. Patients were excluded after enrollment if any of the following occurred: (1) use of antimalarial drugs outside of the study protocol; (2) parasitaemia in the presence of a concomitant febrile illness; (3) withdrawal of consent; (4) loss to follow-up, (5) protocol violation, or (6) death due to a non-malaria illness.

\section{Laboratory procedures}

Blood smears were stained with $4 \%$ Giemsa for $20 \mathrm{~min}$. Parasite densities were determined from thick blood smears by counting the number of asexual parasites per 200 WBCs (or per 500, if the count was less than 10 parasites/200 WBCs), assuming a WBC count of $8,000 / \mu \mathrm{l}$. A smear was considered negative if no parasites were seen after review of 100 fields. Thin blood smears were used to detect non-falciparum infections. Gametocytes were counted against $500 \mathrm{WBC}$ for 287 children at enrolment and in 258,257,220, 217 specimens available for that purpose collected on day 7, day 14, day 21 and day 28, respectively. A portable spectrophotometer (HemoCue ${ }^{\oplus}$, Anglholm, Sweden) was used for haemoglobin assays.

Molecular genotyping techniques were used to distinguish recrudescence from new infection for all patients failing therapy after day 7. Briefly, filter paper blood samples collected on the day of enrollment, on day 1 and on the day of failure were analyzed for polymorphisms in the genes for merozoite surface protein-1 (MSP-1) and merozoite surface protein-2 (MSP-2) using nested-PCR as previously described [12]. First, MSP-2 genotyping patterns on the day of failure were compared with those at treatment initiation and on day 1, using Quantity One $e^{\oplus}$ software (BioRad laboratories, Inc., 1000 Alfred Nobel Drive, Hercules, CA 94547, United States). If all of the MSP-2 alleles present on the day of failure were present at the time of treatment initiation or on day 1, genotyping was repeated using MSP-1. An outcome was defined as recrudescence if all MSP-1 and MSP-2 alleles present at the time of failure were present at the time of treatment initiation or on day 1 , and defined as a new infection otherwise.

\section{Statistical analysis}

Data were entered and verified using EpiInfo $6.04^{\circ}$ software (Centers for Disease Control and Prevention, Atlanta, Georgia, United States), and analysed using MedCalc $^{\bullet}$ software version 9.1.0.1 (MedCalc Software, Broekstraat 52, 9030 Mariakerke, Belgium).

Analysis of treatment outcome was per protocol, which only included patients with treatment outcomes. Frequencies were compared by chi-squared tests and Fisher exact tests, and continuous variables by Student's t-tests, Mann-Whitney U-tests, analysis of variance or KruskalWallis tests as applicable. All reported p-values are twosided, without adjustment for multiple testing, and were considered statistically significant if less than 0.05 .

\section{Results}

695 patients were screened for inclusion in the study and 287 patients were randomized to receive $C Q, A Q, S P, A Q$ + SP or AT + AQ (Figure 1). The baseline characteristics of the patients assigned to the five treatment regimes were similar (Table 1).

Clinical and parasitological monitoring was complete for $96.1 \%(276 / 287)$ until day 14 of follow-up and for $92.3 \%$ (265/287) until day 28. Seventeen patients (5.7\%) were lost to follow-up, four patients withdrew consent (1.3\%) and one patient $(0.3 \%)$ had to be withdrawn because he was treated outside the study with drugs active against malaria (Figure 1).

No severe side-effects attributable to the study medication were observed during the follow-up period, except that one patient treated with $\mathrm{AQ}+\mathrm{SP}$ developed vomiting.

The efficacy of test drugs against uncomplicated falciparum malaria is shown in Table 2. All treatment regimens, except for the CQ treatment group, resulted in 
Table I: Baseline characteristics of patients

\begin{tabular}{|c|c|c|c|c|c|}
\hline \multirow[b]{2}{*}{ Parameters } & \multicolumn{5}{|l|}{ Treatment group } \\
\hline & CQ & AQ & SP & AQ+SP & AQ+AS \\
\hline No. of patients & 42 & 39 & 40 & 83 & 83 \\
\hline No. females (\%) & $20(47.6 \%)$ & $22(56.4 \%)$ & $26(65.0 \%)$ & $42(50.6 \%)$ & $38(46.3 \%)$ \\
\hline Mean age (months. range) & $3.8(0.6-14)$ & $3.3(0.7-13)$ & $3.8(0.5-12)$ & $3.9(0.8-15)$ & $3.9(0.5-15)$ \\
\hline Mean weight (kg. range) & $12.9(7-35)$ & $13.0(7.8-40)$ & $12.1(6-34)$ & $14.3(7-50)$ & $14.6(6-54.5)$ \\
\hline Mean temperature $\left({ }^{\circ} \mathrm{C}\right.$. range $)$ & $38.4(37.5-40.4)$ & $38.4(37.5-40.8)$ & $38.5(37.5-40.8)$ & $38.6(37.5-41.0)$ & $38.6(37.5-40.9)$ \\
\hline 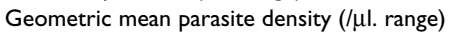 & $21156(1020-199058)$ & $25559(1142-199800)$ & $25640(1010-199531)$ & $22368(1050-199048)$ & $22905(1005-184881)$ \\
\hline Previous antimalrial therapy (\%) & $2(4.7 \%)$ & I (2.9\%) & $2(5.0 \%)$ & $2(3.9 \%)$ & $3(5.3 \%)$ \\
\hline Mean haemoglobin (g/dl. range) & $10.1(6.2-14.0)$ & $10.0(6.4-14.2)$ & $9.7(6.2-12.4)$ & $10.2(6.2-15.6)$ & $10.4(6.2-15.5)$ \\
\hline
\end{tabular}

CQ. Chloroquine; AQ. Amodiaquine; SP. Sulphadoxine-pyrimethamine; AS. artesunate.

clinical cure rates above $97 \%$ by day-14 and $92 \%$ by day28 (PCR-corrected). ETF was significantly less common in the two combinatory treatment $(\mathrm{AQ}+\mathrm{SP}$ and $\mathrm{AQ}+\mathrm{AS})$ groups than in the CQ group on day 14 and day 28 (PCRcorrected and -uncorrected); LCF was significantly less common in $\mathrm{AQ}, \mathrm{SP}, \mathrm{AQ}+\mathrm{SP}$ and $\mathrm{AQ}+\mathrm{AS}$ treatment groups on day 14 and in SP, AQ+SP and AQ+AS on day 28 (PCRuncorrected) and in only the two combinatory treatments on day-28 (PCR-corrected); ACPR to AQ, SP, AQ+SP and AQ+AS did not differ significantly on day-14 or day-28 (PCR-uncorrected and-corrected).

The proportion of re-infections among recurring parasitaemia was higher with CQ $(11.1 \% ; 4 / 36)$ and AQ $(11.1 \% ; 4 / 36)$ than with AQ+SP $(1.2 \% ; 1 / 79 ; \mathrm{P}=0.04)$ (Table 2).
Parasite clearance was more effective with $\mathrm{AQ}+\mathrm{AS}$ than $\mathrm{CQ}, \mathrm{AQ}$ or $\mathrm{AQ}+\mathrm{SP}$ and with SP than CQ until day 2, but less effective with $\mathrm{AQ}+\mathrm{AS}$ than $\mathrm{AQ}+\mathrm{SP}$ on day 28 (Figure 2).

Fever clearance was delayed with SP alone, the proportion of febrile children being significantly lower with $A Q+A S$ and AQ+SP until day-2 and day-1, respectively (Figures 2 and 3).

On day-28, the extent of haematological recovery (median of individual increases in $\mathrm{Hb}$ ) did not differ significantly between the five groups (CQ, $0.8 \mathrm{~g} / \mathrm{dl},-0.8$ to 3.4; $\mathrm{SP}, 1.2 \mathrm{~g} / \mathrm{dl},-1.0$ to $5.8 ; \mathrm{AQ}, 0,9 \mathrm{~g} / \mathrm{dl},-3.6$ to 4.1 ; $\mathrm{AQ}+\mathrm{SP}, 0.5 \mathrm{~g} / \mathrm{dl},-4.4$ to $5.8 ; \mathrm{AQ}+\mathrm{AS}, 1.1 \mathrm{~g} / \mathrm{dl},-2.6$ to 5.2 ).

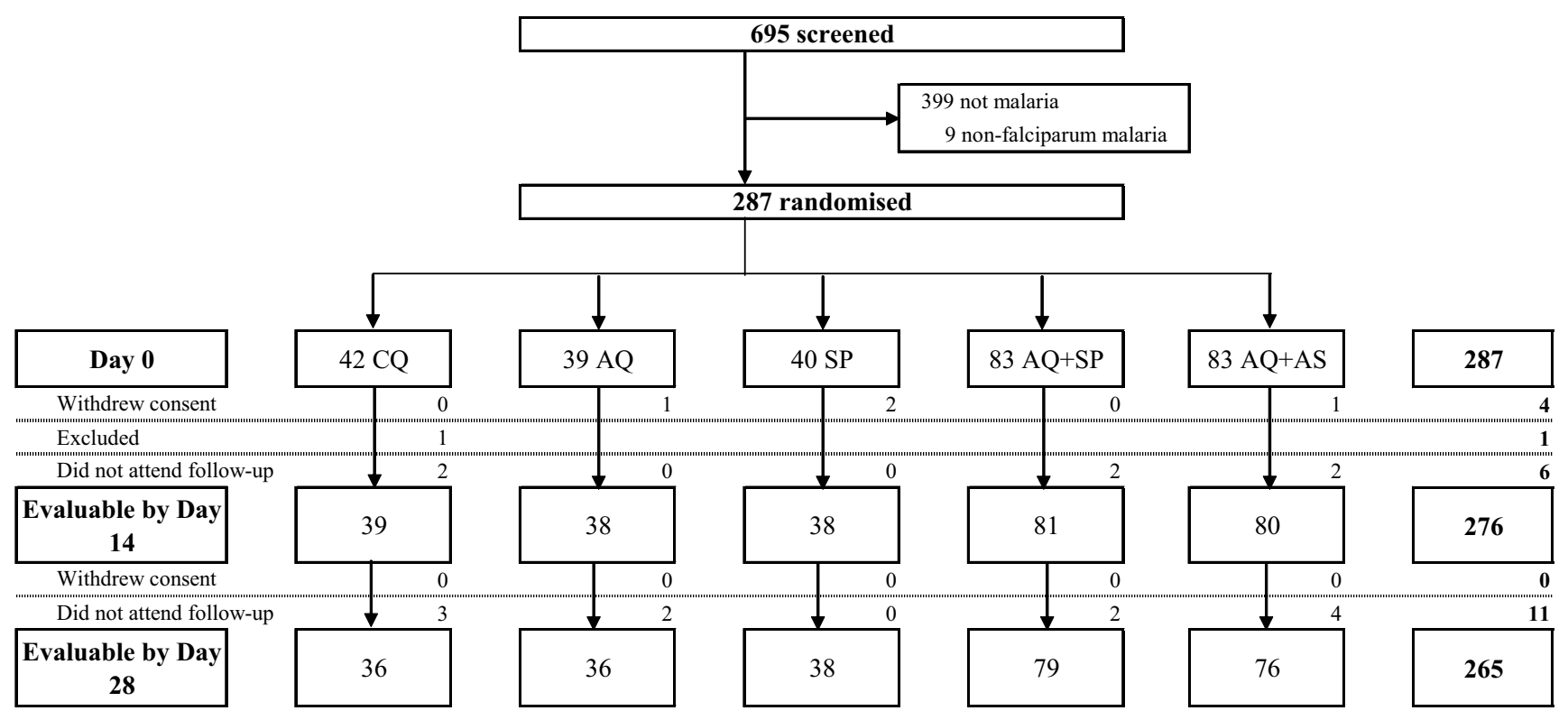

Figure I

Trial profile. 
Table 2: Classification of treatment outcome

\begin{tabular}{|c|c|c|c|c|c|c|c|c|c|c|c|}
\hline \multirow{2}{*}{\multicolumn{2}{|c|}{$\begin{array}{l}\text { Treatment } \\
\text { outcome }\end{array}$}} & \multicolumn{10}{|c|}{ Treatment group } \\
\hline & & \multicolumn{2}{|l|}{ CQ } & \multicolumn{2}{|l|}{ AQ } & \multicolumn{2}{|l|}{ SP } & \multicolumn{2}{|l|}{ AQ+SP } & \multicolumn{2}{|l|}{$A Q+A S$} \\
\hline \multicolumn{12}{|l|}{ I4-day follow-up } \\
\hline ETF (\%) & & $4 / 39(10.3)$ & & $0 / 38(0)$ & & $0 / 38(0)$ & & $0 / 8 \mathrm{I}(0)^{*}$ & & $0 / 80(0)^{*}$ & \\
\hline $\operatorname{LCF}(\%)$ & OTF (\%) & $7 / 39(17.9)$ & $\begin{array}{l}14 / 39 \\
(35.9)\end{array}$ & $0 / 38(0)^{*}$ & $\begin{array}{l}0 / 38 \\
(0)\end{array}$ & $0 / 38(0)^{*}$ & $\begin{array}{l}1 / 38 \\
(2.6)\end{array}$ & $0 / 81(0)^{*}$ & $\begin{array}{l}1 / 81 \\
(1.2)\end{array}$ & $0 / 80(0)^{*}$ & $0 / 80(0)$ \\
\hline LPF (\%) & & $3 / 39(7.7)$ & & $0 / 38(0)$ & & I/38 (2.6) & & $\mathrm{I} / 8 \mathrm{I}(1.2)$ & & $0 / 80(0)$ & \\
\hline ACPR (\%) & & $25 / 39(64.1)$ & & $38 / 38(100)^{*}$ & & $37 / 38(97.4)^{*}$ & & $80 / 81(98.8) *$ & & $80 / 80(100)^{*}$ & \\
\hline \multicolumn{12}{|c|}{ 28-day follow-up non adjusted (PCR-uncorrected) } \\
\hline ETF $(\%)$ & & $4 / 36(11.1)$ & & $0 / 36(0)$ & & $0 / 38(0)$ & & $0 / 79(0)^{*}$ & & $0 / 76(0)^{*}$ & \\
\hline $\operatorname{LCF}(\%)$ & OTF (\%) & $9 / 36(25.0)$ & $\begin{array}{l}20 / 36 \\
(55.5)\end{array}$ & $3 / 36(8.3)$ & $\begin{array}{l}5 / 36 \\
(13.9)\end{array}$ & $0 / 38(0)^{*}$ & $\begin{array}{l}2 / 38 \\
(5.2)\end{array}$ & $2 / 79(2.5)^{*}$ & $\begin{array}{l}4 / 79 \\
(5.0)\end{array}$ & $3 / 76(3.9)^{*}$ & $\begin{array}{l}12 / 76 \\
(15.7)\end{array}$ \\
\hline LPF (\%) & & $7 / 36(19.4)$ & & $2 / 36(5.6)$ & & $2 / 38(5.2)$ & & $2 / 79(2.5)^{*}$ & & $9 / 76(11.8)$ & \\
\hline ACPR (\%) & & $16 / 36(44.5)$ & & $31 / 36(86.1)^{*}$ & & $36 / 38(94.8)^{*}$ & & $75 / 79(95.0)^{*}$ & & $64 / 76(84.3)^{*}$ & \\
\hline \multicolumn{12}{|c|}{ 28-day follow-up adjusted (PCR-corrected) } \\
\hline New infections (\%) & & $4 / 36(11.1)$ & & $4 / 36(11.1)$ & & I/38 (2.6) & & $1 / 79(1.2)^{* * *}$ & & $6 / 76(7.9)$ & \\
\hline ETF $(\%)$ & & $4 / 36(11.1)$ & & $0 / 36(0)$ & & $0 / 38(0)$ & & $0 / 79(0)^{*}$ & & $0 / 76(0)^{*}$ & \\
\hline LCF (\%) & OTF (\%) & $7 / 36(19.4)$ & $\begin{array}{l}13 / 36 \\
(44.4)\end{array}$ & I/36 (2.8) & $\begin{array}{l}1 / 36 \\
(2.8)\end{array}$ & $0 / 38(0)$ & $\begin{array}{l}1 / 38 \\
(2.6)\end{array}$ & $\mathrm{I} / 79(1.3)^{*}$ & $\begin{array}{l}3 / 78 \\
(3.8)\end{array}$ & $\mathrm{I} / 76(1.3)^{*}$ & $6 / 76(7.9)$ \\
\hline LPF (\%) & & $5 / 36(13.9)$ & & $0 / 36(0)^{*}$ & & I/38 (2.6) & & $2 / 79(2.5)^{*}$ & & $5 / 76(6.6)$ & \\
\hline ACPR (\%) & & $20 / 36(55.6)$ & & $35 / 36(97.2)^{*}$ & & $37 / 38(97.4)^{*}$ & & $76 / 79(96.2)^{*}$ & & $70 / 76(92.1)^{*}$ & \\
\hline
\end{tabular}

Data presented are cumulative, failures up to day 14 of follow-up are also considered in the calculation of day 28 failure rates.

Percentages are rounded to achieve totals of $100 \%$.

$\mathrm{CQ}$, Chloroquine; AQ, amodiaquine; SP, Sulphadoxine-pyrimethamine; AS, artesunate ETF, early treatment failure; LCF, late clinical failure; LPF, late parasitological failure; $A C P R$, adequate clinical and parasitological response.

* Significant difference to CQ group $(P<0.05)$; ** Significant difference to $C Q$ and $A Q$ groups $(P<0.05)$.

Gametocyte prevalence at enrolment was 5.6\% (16/287) with a geometric mean density of 124.7 gametocytes/ $\mu$ l (95\% confidence interval, 50.5-307.9). There were no significant differences at enrolment in gametocyte prevalence or density with respect to treatment regime (Table

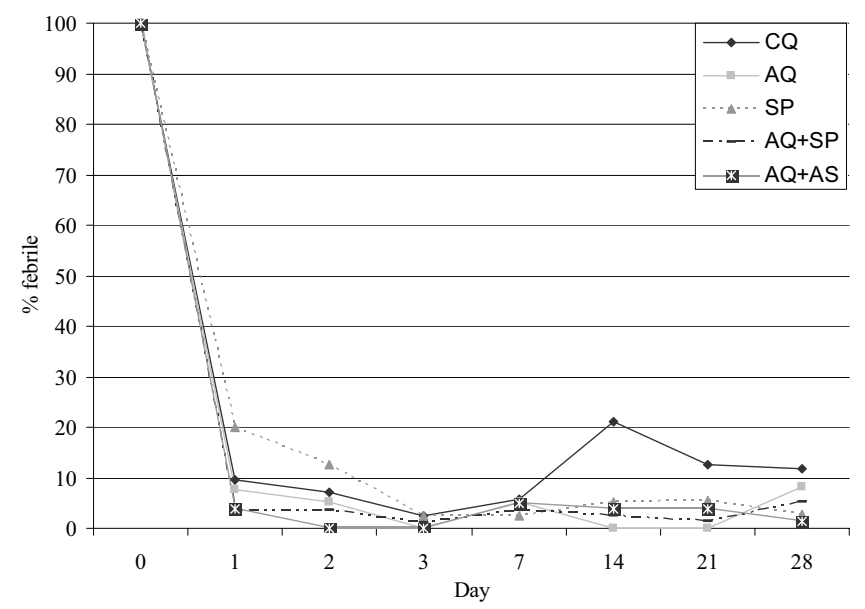

Figure 2

Proportions of febrile patients following treatment. $\mathrm{CQ}$, Chloroquine, AQ, Amodiaquine; SP, SulfadoxinePyrimethamine; AS, artesunate; On day I, Significant difference to $S P$ group $(P<0.05)$, SP vs. $A Q+S P, S P$ vs. $A Q+A S$; At day 2 , Significant difference to $S P$ group $(P<0.05)$, SP vs. $A Q+A S ;$ On day I4, Significant difference to $C Q$ group $(P<$ $0.05), C Q$ vs. $A Q, C Q$ vs. $S P, C Q$ vs. $A Q+S P, C Q$ vs. $\mathrm{AQ}+\mathrm{AS}$
3). Treatment with SP gave significantly higher gametocyte prevalence on day- 7 and on day-14 than the other treatments. On day-21, gametocyte prevalence in SP group was significantly higher than the two combinatory treatments $(\mathrm{AQ}+\mathrm{SP}$ and $\mathrm{AQ}+\mathrm{AS})$.

\section{Discussion}

The main objective of this study was to compare, for the first time in Madagascar, the efficacy of two different combination therapies: $\mathrm{AQ}+\mathrm{SP}$, an inexpensive regimen that has proven to be efficacious in recent studies [13-16] and AQ+AS, an ACT regimen chosen by National Malaria Control Programme (NMCP) as first-line treatment for uncomplicated malaria. This clinical trial was designed to include follow-up for children in each treatment group enrolled within 28 days because it had been shown that antimalarial drug efficacy studies that limit follow-up to 14 days or less may significantly underestimate the risk of re-emergence [17].

The findings are the preliminary results of an extended study of the efficacy of antimalarial drugs for the treatment of uncomplicated falciparum malaria, which involves eight sites with differing levels of transmission intensity across Madagascar. The methodology used was based on the 2003 WHO protocol [11], with some modifications (compromise between the high transmission and the low to moderate transmission protocols): (i) children enrolled in the study were between 6 months and 15 years of age and (ii) of $P$. falciparum parasitaemia on inclusion was between 1,000 and 200,000/ $\mu$ l. 


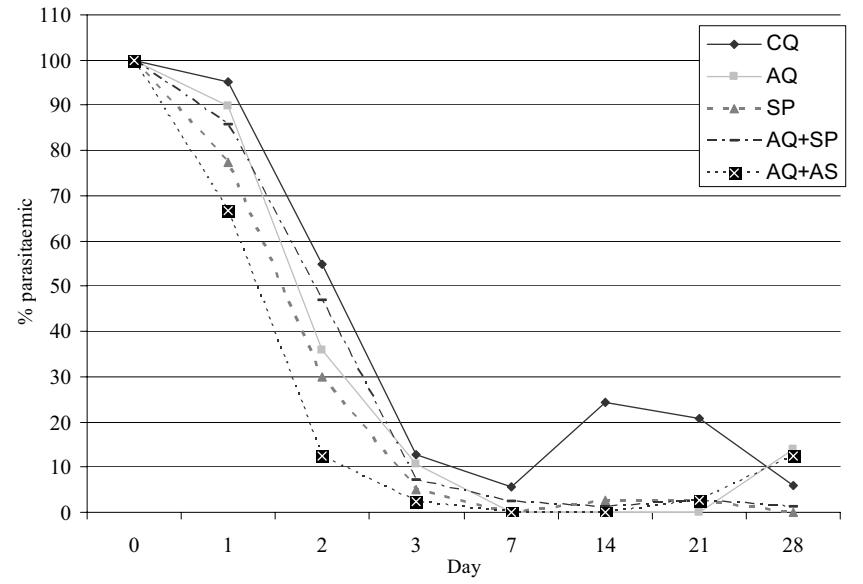

Figure 3

Proportions of parasitaemic patients following treatment. CQ, Chloroquine, AQ, Amodiaquine; SP, SulfadoxinePyrimethamine; AS, artesunate; On day I, Significant difference to $C Q$ group $(P<0.05), C Q$ vs. $S P, C Q$ vs. $A Q+A S$; Significant difference to $A Q$ group $(P<0.05), A Q$ vs. $A Q+A S$; Significant difference to $A Q+S P$ group $(P<0.05)$, $A Q+S P$ vs. $A Q+A S ; O n$ day 2 , Significant difference to $S P$ group $(P<0.05)$, SP vs. $C Q$; Significant difference to $A Q+A S$ group $(P<0.05), A Q+A S$ vs. $C Q, A Q+A S$ vs. $A Q, A Q+A S$ vs. SP, AQ+AS vs. AQ+SP; on day I4, Significant difference to $C Q$ group $(P<0.05), C Q$ vs. $A Q, C Q$ vs. $S P, C Q$ vs. $A Q+S P, C Q$ vs. $A Q+A S$; On day 21 , Significant difference to $C Q$ group $(P<0.05), C Q$ vs. $A Q, C Q$ vs. $A Q+S P, C Q$ vs. $A Q+A S ;$ On day 28, Significant difference to $A Q+S P$ group $(P$ $<0.05), A Q+S P$ vs. $A Q+A S$

The first step was to update data concerning therapeutic efficacies of CQ, AQ and SP alone, because CQ is has long been widely used in Madagascar and recommended by the NMCP for the home management of presumed malaria in children under five years of age (HMM). Prepackaged presentations of chloroquine are currently available for children from six to 11 months of age and for chil- dren from 12 to 59 months of age and either sold at an affordable price of US $\$ 0.025$ (PaluStop ${ }^{\circledR}$ ) or freely distributed at primary public health facilities (Ody Tazomoka ${ }^{\circledR}$ ) [18]. SP has also been used, since 2005, for intermittent preventive treatment in pregnant women in all areas of Madagascar except the Central Highlands.

No severe side-effects attributable to study medication were found during the follow-up period in any of the treatment groups. CQ was significantly less effective than $\mathrm{SP}, \mathrm{AQ}, \mathrm{AQ}+\mathrm{SP}$ and $\mathrm{AQ}+\mathrm{AS}$ in treating uncomplicated falciparum malaria, with overall treatment failure of $35.9 \%$ within 14 days of follow up and $44.4 \%$ within 28 days (PCR-corrected). These data show a higher prevalence of chloroquine resistance than reported in previous studies [19-21] and a good effectiveness of SP and AQ, as had been observed in many areas in West Africa [22,23]. These results confirm the urgent need to replace pre-packaged CQ with another pre-packaged effective therapy. According to the NMCP of the Ministry of Health of Madagascar, this switch will be gradual with the introduction of prepackaged artemisinin-based combination therapies (coformulated presentation of the $A Q+A S$ combination, COARSUCAM $^{\circ}$, Sanofi-Synthélabo Groupe, Paris, France) from the beginning of 2008.

This study also confirms that the assumption that ACTs are always more effective than NACTs is not always true especially in settings, such as Madagascar, where SP or AQ monotherapies remain effective. It was observed that $\mathrm{AQ}+\mathrm{SP}$ was as effective as $\mathrm{AQ}+\mathrm{AS}$, consistent with previous reports in Africa both in high transmission $[14,15,24]$ and in low transmission areas [16]. No significant difference between the two combination treatments was observed in terms of efficacy, safety, tolerance, fever clearance, or haematological recovery. Moreover, no significant difference in gametocyte prevalence was found between the two combination treatments. The observed lower prevalence of gametocytes in $A Q+S P$ and $A Q+A S$

Table 3: Gametocyte prevalence on days $0,7,14,21$ and 28 after treatment in patients with adequate parasitological response

Gametocyte prevalence, \%, (n/N)

\begin{tabular}{llllll}
\cline { 3 - 5 } & \multicolumn{3}{c}{ After treatment } & & \\
\cline { 3 - 6 } & At enrolment & Day 7 & Day I4 & Day 2 I & Day 28 \\
\hline CQ & $4.7(2 / 42)$ & $12.5(3 / 24)^{*}$ & $4.2(1 / 24)^{*}$ & $6.6(1 / 15)$ & $0(0 / 15)$ \\
AQ & $12.8(5 / 39)$ & $13.1(5 / 38)^{*}$ & $10.5(4 / 38)^{*}$ & $9.6(3 / 31)$ & $0(0 / 30)$ \\
SP & $10.2(4 / 39)$ & $44.4(16 / 36)$ & $33.3(12 / 36)$ & $17.1(6 / 35)$ & $6.1(2 / 33)$ \\
AQ+SP & $2.4(2 / 83)$ & $3.8(3 / 80)^{*}$ & $6.3(5 / 80)^{*}$ & $1.3(1 / 75)^{\pi}$ & $1.3(1 / 75)$ \\
AQ+AS & $3.6(3 / 83)$ & $2.5(2 / 80)^{*}$ & $1.2(1 / 79)^{*}$ & $1.5(1 / 64)^{\pi}$ & $0(0 / 64)$ \\
\hline
\end{tabular}

$\mathrm{CQ}$, Chloroquine, $\mathrm{AQ}$, amodiaquine; SP, Sulphadoxine-Pyrimethamine; $\mathrm{AS}$, artesunate; $95 \% \mathrm{Cl}, 95 \%$ confidence interval.

* On day 7 and day 14 , significant difference to SP group $(P<0.05)$

TOn day 21 , significant difference to SP group $(P<0.05)$ 
groups reflect the effect of the additional drug on gametocyte survival or development, as has been previously shown for $A Q+A S[14]$ and $A Q+S P[9,25]$.

Although AQ+AS produced faster parasite clearance than $A Q+S P$, the risk of new infection within the month after therapy was high with $A Q+A S$ than $A Q+S P$ treatment, even in a low transmission area such as Moramanga. This phenomenon has previously been observed in areas of high transmission [14,24]. According to Krishna [26] and Watkins [27], the prevention of new infections by AQ+SP is probably due to the long elimination half-lives of the two drugs, whereas with $\mathrm{AQ}+\mathrm{AS}$ combination, $\mathrm{AS}$ is rapidly eliminated, leaving only AQ to provide post-treatment prophylaxis.

However, cost and availability of ACTs remain major concerns, and it appears that the sudden increase in demand for artemisinins may exacerbate these problems, at least in the short-term [7]. AQ+AS (0.51 US\$) [28] is currently four times more expensive than $A Q+S P$ (0.13 US\$). In Madagascar, with a reported 2,114,400 cases of suspected malaria (2003), the use of $A Q+S P$ instead of $A Q+A S$ would reduce the annual antimalarial treatments costs by 800,000 US $\$$. In view of the recent report by Tagbor and colleagues [29] there is hope that AQ+SP could serve as a safe and effective alternative for malaria treatment in pregnancy and for intermittent preventive treatment until the safety of ACTs for pregnant women has been established. Moreover, further studies should be carry out to assess the usefulness of $\mathrm{AQ}+\mathrm{SP}$ pre-packed fixed-dose combination as alternative of SP alone in intermittent preventive treatment in infants and as alternative for CQ for the home management of presumed malaria in children under five years of age.

In conclusion, these results indicate that $A Q+S P$ is as effective as $A Q+A S$, and thereby show that the inexpensive and widely available combination $\mathrm{AQ}+\mathrm{SP}$ may still be appropriate for the treatment of uncomplicated malaria in Madagascar, an area where resistance to the drugs is relatively uncommon. This could have an important role in this country, where diagnostic services are not accurate and where much of the drugs administered go to patients who do not have malaria.

\section{Authors' contributions}

Didier Ménard was involved in all stages of this study. Arsène Ratsimbasoa, Nohary Nina Harimanana Andrianina, Zakaherizo Ramiandrasoa, Arthur Randriamanantena and Noéline Rasoarilalao performed the field work. Martial Jahevitra performed molecular genotyping. Luciano Tuseo and Andrianirina Raveloson helped to compose the manuscript and gave constructive advice.

\section{Acknowledgements}

We thank all the patients and their parents or guardians for participating in the study.

This work was supported by Natixis, the Global Fund to Fight AIDS, Tuberculosis and Malaria round 3 grant MDG-304-G05-M and the IAEA project, RAF-6-025-9002.

\section{References}

I. Snow RW, Guerra CA, Noor AM, Myint HY, Hay SI: The global distribution of clinical episodes of Plasmodium falciparum malaria. Nature 2005, 434:2I4-217.

2. World Health Organization: Implementation of the Global Malaria Control Strategy. Report of a WHO Study Group on the implementation of the global plan of action for malaria control, 1993-2000. World Health Organization, Geneva, Technical Report Series No 8391993.

3. Attaran A, Barnes KI, Curtis C, d'Alessandro U, Fanello $\mathrm{Cl}$, Galinski MR, Kokwaro G, Looareesuwan S, Makanga M, Mutabingwa TK, Talisuna A, Trape JF, Watkins WM: WHO, the Global Fund, and medical malpractice in malaria treatment. Lancet 2004, 363:237-240.

4. White NJ: Antimalarial drug resistance. J Clin Invest 2004 , I 1 3: 1084-1092

5. Kremsner PG, Krishna S: Antimalarial combinations. Lancet 2004 , 364:285-294.

6. World Health Organization: The use of artemisinin and its derivatives as antimalarial drugs: a report of a joint CTD/DMP/ TDR Informal Consultation. World Health Organization, Geneva, WHOIMAL/981086 1998

7. Snow RW, Eckert E, Teklehaimanot A: Estimating the needs for artesunate-based combination therapy for malaria casemanagement in Africa. Trends Parasitol 2003, 19:363-369.

8. World Health Organization: Monitoring Antimalarial Drug Resistance. 2001 , WHO/CDS/CSR/EPH/ 2002.7.Geneva:WHO:

9. Dorsey G, Njama D, Kamya MR, Cattamanchi A, Kyabayinze D, Staedke SG, Gasasira A, Rosenthal PJ: Sulphadoxine/pyrimethamine alone or with amodiaquine or artesunate for treatment of uncomplicated malaria: a longitudinal randomised trial. Lancet 2002, 360:203I-2038.

10. Rwagacondo CE, Niyitegeka F, Sarushi J, Karema C, Mugisha V, Dujardin JC, Van Overmeir C, Van Den Ende J, d'Alessandro U: Efficacy of amodiaquine alone and combined with sulphadoxinepyrimethamine and of sulphadoxine-pyrimethamine combined with artesunate. Am J Trop Med Hyg 2003, 68:743-747.

II. World Health Organization: Assessment and monitoring of antimalarial drug efficacy for the treatment of uncomplicated falciparum malaria. Geneva: WHO 2003.

12. Cattamanchi A, Kyabayinze D, Hubbard A, Rosenthal PJ, Dorsey G Distinguishing recrudescence from reinfection in a longitudinal antimalarial drug-efficacy study: Comparison of results based on genotyping of $\mathbf{m s p}-\mathrm{I}, \mathbf{m s p}-\mathbf{2}$, and glurp. Am J Trop Med Hyg 2003, 68:133-139.

13. Hwang J, Bitarakwate E, Pai M, Reingold A, Rosenthal PJ, Dorsey G: Chloroquine or amodiaquine combined with sulfadoxinepyrimethamine for uncomplicated malaria: a systematic review. Trop Med Int Health 2006, I I (6):789-799.

14. Yeka A, Banek K, Bakyaita N, Staedke SG, Kamya MR, Talisuna A Kironde F, Nsobya SL, Kilian A, Slater M, Reingold A, Rosenthal PJ, Wabwire-Mangen F, Dorsey G: Artemisinin versus nonartemisinin combination therapy for uncomplicated malaria: randomized clinical trials from four sites in Uganda. PLoS Med 2005, 2(7): 190.

15. Obonyo CO, Juma EA, Ogutu BR, Vulule JM, Lau J: Amodiaquine combined with sulphadoxine/pyrimethamine versus artem. isinin-based combinations for the treatment of uncomplicated falciparum malaria in Africa: a meta-analysis. Trans $R$ Soc Trop Med Hyg 2007, I OI: I 17-1 26.

16. Staedke SG, Mpimbaza A, Kamya MR, Nzarubara BK, Dorsey G, Rosenthal PJ: Combination treatments for uncomplicated falciparum malaria in Kampala, Uganda: Randomised clinical trial. Lancet 2004, 364:1950-1957. 
17. White $\mathrm{N}$ : The assessment of antimalarial drug efficacy. Trends Parasitol 2002, 18:458-464.

18. Ratsimbasoa A, Randrianarivelojosia M, Millet P, Soares JL, Rabarijaona L, Rakotoson B, Malvy D, Menard D: Use of pre-packaged chloroquine for the home management of presumed malaria in Malagasy children. Malar J 2006, 5:79.

19. Willcox ML, Rakotondrazafy E, Andriamanalimanana R, Andrianasolo $D$, Rasoanaivo P: Decreasing clinical efficacy of chloroquine in Ankazobe, Central Highlands of Madagascar. Trans $R$ Soc Trop Med Hyg 2004, 98:3II-3I4.

20. Raharimalala LA, Rabarijaona L, Randrianarivelojosia M, Razanavololo F, Rason MA, Andrianantenaina HB, Andrianaivolambo L, Rakotoniaina JC, Leong Pock Tsi JM, Rajaonarivelo E, Leon T, Duchemin JB, Ariey F: Malaria study in the cyclone risk zone: entomological , diagnostic and therapeutic approach in the southeastern region of Madagascar. Arch Inst Pasteur Madagascar 2002, 68(I2):79-85.

21. Randrianarivelojosia M, Raharimalala L, Randriamanantena A, Jambou R: Drug resistance of Plasmodium falciparum in coastal regions of Madagascar. Med Trop 2000, 60(3):243-249.

22. Randrianasolo L, Randriamanantena $A$, Ranarivelo $L$, Ratsimbasoa $A$, Domarle O, Randrianarivelojosia M: Monitoring susceptibility to sulphadoxine-pyrimethamine among cases of uncomplicated Plasmodium falciparum malaria in Saharevo, Madagascar. Ann Trop Med Parasitol 2004, 98(6):55 I-554.

23. Randrianasolo L, Randriamanantena A, Ratsimbasoa A, Rakotoson JD, Randriambelosoa J, Raveloson A, Rakotondrajaona N, Tuseo L, Randrianarivelojosia M: Assessment of sulphadoxine-pyrimethamine (Fansidar, Paludar) efficacy in patients with uncomplicated malaria in Madagascar: preliminary study to propose a simplified study protocol. Arch Inst Pasteur Madagascar 2003, 69(I-2):52-56.

24. Mockenhaupt FP, Ehrhardt S, Dzisi SY, Teun Bousema J, Wassilew N, Schreiber J, Anemana SD, Cramer JP, Otchwemah RN, Sauerwein RW, Eggelte TA, Bienzle U: A randomized, placebo-controlled, double-blind trial on sulphadoxine-pyrimethamine alone or combined with artesunate or amodiaquine in uncomplicated malaria. Trop Med Int Health 2005, I 0(6):5। 2-520.

25. Schellenberg D, Kahigwa E, Drakeley C, Malende A, Wigayi J, Msokame C, Aponte J], Tanner M, Mshinda H, Menendez C, Alonso PL: The safety and efficacy of sulphadoxine/pyrimethamine, amodiaquine, and their combination in the treatment of uncomplicated Plasmodium falciparum malaria. Am J Trop Med Hyg 2002, 67:17-23.

26. Krishna S, White NJ: Pharmacokinetics of quinine, chloroquine and amodiaquine. Clinical implications. Clin Pharmacokinet 1996, 30:263-299.

27. Watkins WM, Mosobo M: Treatment of Plasmodium falciparum malaria with pyrimethamine/sulphadoxine: Selective pressure for resistance is a function of long elimination halflife. Trans R Soc Trop Med Hyg 1993, 87:75-78.

28. Wiseman V, Kim M, Mutabingwa TK, Whitty CJ: Cost-Effectiveness Study of Three Antimalarial Drug Combinations in Tanzania. PLoS Med 2006, 3(10):.

29. Tagbor H, Bruce J, Browne E, Randal A, Greenwood B, Chandramohan D: Efficacy, safety, and tolerability of amodiaquine plus sulphadoxine-pyrimethamine used alone or in combination for malaria treatment in pregnancy: a randomised trial. Lancet 2006, 368(9544): 1349-1356.
Publish with Biomed Central and every scientist can read your work free of charge

"BioMed Central will be the most significant development for disseminating the results of biomedical research in our lifetime. "

Sir Paul Nurse, Cancer Research UK

Your research papers will be:

- available free of charge to the entire biomedical community

- peer reviewed and published immediately upon acceptance

- cited in PubMed and archived on PubMed Central

- yours - you keep the copyright

Submit your manuscript here:

http://www.biomedcentral.com/info/publishing_adv.asp
BiolMedcentral 